\title{
THE NEARBY YOUNG DWARF GALAXY SBS 0335-052: PRIMORDIAL GAS AND LYMAN-ALPHA EMISSION
}

\author{
TRINH XUAN THUAN AND YURI I. IZOTOV \\ Astronomy Department, University of Virginia \\ Charlottesville, VA 22903, USA
}

AND

Main Astronomical Observatory, Kiev 252650, Ukraine

Ever since their discovery, blue compact dwarf (BCD) galaxies have been thought of as excellent candidates for being young galaxies, i.e. systems presently undergoing one of their very first bursts of star formation. This is mainly because BCDs are very metal-deficient, the metallicities of their ionized gas ranging between $Z_{\odot} / 50$ and $Z_{\odot} / 3$, which makes them the least chemically evolved galaxies in the Universe. Other evidence, such as the very high fractional neutral hydrogen gas content and the lack of an evident underlying old stellar population on optical images, also point to the relative youth of some BCDs.

Thuan, Izotov \& Lipovetsky (1997) have argued SBS 0335-052 to be a young galaxy on the basis of the following evidence: 1) HST imaging of the BCD shows its underlying extended low surface brightness component to have an irregular and filamentary structure, suggesting that a significant part of the emission $(\sim 1 / 3)$ comes not from an underlying stellar population, but from ionized gas. Any underlying stellar population must be younger than $\sim 10^{8}$ yr. Propagating star formation occurs in a chain of 6 super-star clusters with ages ranging between 4 and $30 \mathrm{Myr}$. 2) The underlying component shows unusually blue colors consistent with gaseous emission, in contrast to most BCDs which possess an underlying red component. 3) VLA $21 \mathrm{~cm}$ observations show the BCD to be embedded in an extraordinarily large HI cloud with dimensions some $64 \times 24$ $\mathrm{kpc}$ (the typical size of $\mathrm{HI}$ envelopes around BCDs is more like a few kpc in each dimension).

HST UV spectrophotometry of SBS 0335-052 by Thuan \& Izotov (1997) shows a broad damped Lyo absorption giving a HI column density of $\mathrm{N}(\mathrm{HI})=(7.0 \pm 0.5) \times 10^{21} \mathrm{~cm}^{-2}$, one of the largest HI column densities known in a galaxy. The UV spectrum also shows many absorption lines of heavy elements, such as O I $\lambda 1302$, Si II $\lambda 1260, \lambda 1304$, and S II $\lambda 1251, \lambda 1254, \lambda 1259$, at the redshift of the BCD. Comparison with high resolution quasar spectra suggests that the O I $\lambda 1302$, Si II $\lambda 1304$ and S II $\lambda 1251, \lambda 1254$ absorption lines are probably not saturated and can be used to derive column densities. If these lines originate from the neutral gas envelope of SBS 0335-052, then the abundances of oxygen, silicon and sulfur are respectively 37000,4000 and 116 times lower than the solar values. These highly discrepant deficiency factors suggest that the heavy element absorption lines are produced not in the H I, but in the H II region. If so, oxygen and sulfur abundances can be derived from the absorption lines which are in agreement, within a factor of 2 , with those derived from the optical emission lines, giving a metallicity $\sim Z_{\odot} / 40$ for the HII region. If the heavy element absorption lines all come from the H II region, then the HI cloud in SBS 0335-052 is primordial, composed of pristine gas unenriched in heavy elements by previous star formation.

How do the properties of SBS 0335-052 compare with those of young, high-redshift galaxies? The star formation rate $(S F R)$ in the BCD is moderate, equal only to $\sim 0.5 M_{\odot \mathrm{yr}^{-1}}$, about one order of magnitude lower than the $S F R$ s derived by Steidel et al. (1996) for their high-redshift star-forming galaxies. SBS 0335-052 contains an equivalent number of $\sim 4500 \mathrm{O} 7 \mathrm{stars,} \mathrm{so} \mathrm{that} \mathrm{its}$ ultraviolet luminosity is $\sim 10^{9} L_{\odot}$, in the low range of ultraviolet luminosities $\left(10^{9}-10^{10} L_{\odot}\right)$ found by Lanzetta et al. (1996) in their study of high-redshift $(2.5 \leq z \leq 6)$ galaxies in the Hubble Deep Field. The sizes of the star-forming regions in these galaxies are $\sim 1 \mathrm{kpc}$, which compare well with that in SBS 0335-052. These luminosities and sizes are modest in comparison to luminous non-dwarf galaxies because star formation in the high-redshift objects occurs in small, concentrated regions 
rather than in galaxy-sized ones. This is in contrast to the situation in a BCD like SBS 0335 -052 where star formation occurs over the whole galaxy. As for the metallicities, the ones deduced for high-redshift systems are generally in the range spanned by BCDs, but with the vast majority of the known systems being significantly more chemically evolved than SBS 0335-052. For example, Pettini et al. (1997) found a mean metallicity of $\sim Z_{\odot} / 13$ for 10 damped Ly $\alpha$ systems in the redshift range $z=0.7$ to 3.4 . Thus, except for their higher star formation rates, the star-forming regions in high-redshift galaxies have properties which are very similar to those in young nearby dwarf galaxies like SBS0335-052. It is still not yet clear why SBS0335-052 did not participate in star formation and metal-enrichment at the same epoch as the majority of the galaxies, given that the collapse time of its $\mathrm{H}$ I envelope is only $\sim 5 \times 10^{8} \mathrm{yr}$, much shorter than the Hubble time.

A most interesting result is the detection in the spectrum of SBS 0335-052 of strong Si IV $\lambda 1394, \lambda 1403$ lines with $\mathrm{P}$ Cygni profiles. A minimum amount of metals is needed to provide the necessary opacity to drive the stellar winds originating from massive $O$ stars which are responsible for the P Cygni profiles. The problem is especially acute in the case of SBS 0335-052 where, as suggested above, the OB stars seen now in the starburst probably formed out of pristine primordial gas, unenriched in heavy elements by previous star formation. Since the present star formation has proceeded in 6 separate bursts of duration less than a few Myr, we can imagine a scenario where the stars born in the later bursts and responsible for the stellar winds, formed from gas already enriched in heavy elements by supernovae resulting from the previous bursts, although it is not clear whether the metals produced in supernovae have had enough time to mix with the pristine gas. Another possibility is to postulate that somehow the evolution of massive stars leads to an increase of their surface metallicity and hence to the onset of a stellar wind.

What are the implications of the non-detection of Ly $\alpha$ emission in SBS 0335-052 concerning the escape of Ly $\alpha$ photons from a galaxy? It has been suggested that there should be an increase in the $\mathrm{Ly} \alpha / \mathrm{H} \beta$ flux ratio as the metallicity of the galaxy decreases, since presumably low metallicity objects contain less dust, and hence suffer less Ly $\alpha$ photon destruction by dust. The observational evidence for such a correlation is mixed. Terlevich et al. (1993) did find one, while Giavalisco et al. (1996) found a very weak one. The fact that there is no Ly $\alpha$ emission in the two most metal-deficient BCDs known, I Zw 18 (Kunth et al. 1994) and SBS 0335-052 argues strongly against the existence of such correlation. Thus metallicity, and hence dust, cannot be the only factor determining the escape of Ly $\alpha$ photons in SBS 0335-052, although HST optical pictures show clear evidence of dust in the BCD (Thuan et al. 1997). Lequeux et al. (1995) have argued that Ly $\alpha$ photons can escape when the neutral material where the absorption occurs is outflowing with respect to the star-forming region. While there is evidence for fast gas motions in SBS 0335-052 with velocities up to $\sim 1500 \mathrm{~km} \mathrm{~s}^{-1}$, the $\mathrm{H}$ I gaseous envelope appears to be static with respect to the $\mathrm{H}$ II region, as the $21 \mathrm{~cm}$ and emission-line velocities are in good agreement. Thus, with its extremely large $\mathrm{H} \mathrm{I}$ column density, the redistribution of Ly $\alpha$ photons in SBS 0335-052 by multiple scattering over the large volume of the $\mathrm{H}$ I cloud probably plays also an important role in diminishing the intensity of the Lya line. The orientation of the flattened H I cloud may also play a role. Because the HI envelope is seen edge-on, Ly $\alpha$ photons escape more easily along directions perpendicular to the line of sight than along it.

\section{References}

Giavalisco, M., Koratkar, A. and Calzetti, D. 1996, ApJ, 461, 831

Kunth, D., Lequenx, J., Sargent, W.L.W. and Viallefond, F. 1994, AA, 282, 709

Lanzetta, K.M., Yahil, A. and Fernandez-Soto, A. 1996, Nature, 381, 759

Lequeux, J., Kunth, D., Mas-Hesse, J.M. and Sargent, W.L.W. 1995, AA, 301, 18

Pettini, M., King, D.L., Smith, L.J. and Hunstead, R.W. 1997, ApJ, 478, 536

Steidel,C.C., Giavalisco, M., Pettini, M., Dickinson, M. and Adeberger, K.L. 1996, ApJ, 462, L17

Terlevich, E., Diaz, A.I., Terlevivh, R. and Garcia Vargas, M.L. 1993, MNRAS, 260, 3

Thuan, T.X. and Izotov, Y.I. 1997, ApJ, November 10

Thuan, T.X., Izotov, Y.I., and Lipovetsky, V.A. 1997, ApJ, 477, 661. 